\title{
EFFECT OF VISCOUS DISSIPATION AND THERMAL RADIATION ON HEAT TRANSFER OVER A NON-LINEARLY STRETCHING SHEET THROUGH POROUS MEDIUM
}

\author{
M.M. NANDEPPANAVAR ${ }^{*}$ and M.N. SIDDALINGAPPA \\ Department of PG and UG Studies in Mathematics \\ Government College \\ Gulbarga-585105 Karnataka, INDIA \\ E-mail:mahantesh_maths@yahoo.co.in
}

\begin{abstract}
In this present paper, we have discussed the effects of viscous dissipation and thermal radiation on heat transfer over a non-linear stretching sheet through a porous medium. Usual similarity transformations are considered to convert the non-linear partial differential equation of motion and heat transfer into ODE's. Solutions of motion and heat transfer are obtained by the Runge-Kutta integration scheme with most efficient shooting technique. The graphical results are presented to interpret various physical parameters of interest. It is found that the velocity profile decreases with an increase of the porous parameter asymptotically. The temperature field decreases with an increase in the parametric values of the Prandtl number and thermal radiation while with an increase in parameters of the Eckert number and porous parameter, the temperature field increases in both PST (power law surface temperature) and PHF (power law heat flux) cases. The numerical values of the non-dimensional wall temperature gradient and wall temperature are tabulated and discussed.
\end{abstract}

Key words: porous media, viscous dissipation, Runge-Kutta method, thermal radiation, wall temperature gradient, wall temperature.

\section{Introduction}

As we know the boundary layer behaviour over a moving continuous solid surface is an important type of flow occurring in several engineering processes. The heat transfer due to a continuously moving stretching surface through an ambient liquid is one of the thrust areas of current research. Such investigations find their application over a broad spectrum of science and engineering disciplines, especially in the field of chemical engineering. Many chemical engineering processes like metallurgical process, polymer extrusion process involve cooling of a molten liquid being stretched into a cooling system. The fluid mechanical properties desired for an outcome of such a process would mainly depend on two aspects, one is the cooling liquid used and the other is the rate of stretching. Liquids of non-Newtonian characteristics, which are electrically conducting, can be opted as a cooling liquid as the flow and the heat transfer can be regulated through some external agency. The rate of stretching is very important as rapid stretching results in sudden solidification, thereby destroying the properties expected for the outcome. The problem mentioned here is a fundamental one and frequently arises in many practical situations such as polymer extrusion processes. It is also encountered in other processes such as drawing, annealing and tinning of copper wires, continuous stretching, rolling and manufacturing of plastic films and artificial fibers, heat treated materials traveling on conveyer belts, glass blowing, crystal growing, paper production and so on.

As the flow through porous media plays an important role in many practical applications such as ground water flows, enhanced oil recovery process, contamination of soils by hazardous wastes, pollution movement, etc., contributions towards the study of stretching sheet problems in a porous medium are numerous.

\footnotetext{
* To whom correspondence should be addressed
} 
Sakiadis (1961) investigated the boundary layer flow over a flat surface moving with a constant velocity and formulated a boundary layer equation for the two dimensional and axisymmetric cases. Crane (1970) studied the steady two dimensional flow caused by the stretching of an elastic flat surface, which moves on its own plane with a velocity varying linearly with distance from a fixed point. Works by Sakiadis (1961) and Crane (1970) were further extended to include the effects of suction/injection, heat transfer, mass transfer, porous medium, magnetic fluid, non-Newtonian fluids, etc.

Gupta and Gupta (1977) studied the effects of surface suction (or injection) on Crane's flow Crane (1970). Carragher and Crane (1982) investigated the heat transfer in a two dimensional flow past a stretching sheet when the temperature difference between the surface and the ambient fluid is proportional to a power of distance from the fixed point. Liu (2005) and Liu (2006) studied the heat and mass transfer problems for a viscous fluid-saturated porous medium over an impermeable/permeable and non-isothermal stretching sheet under various environment, respectively. The steady and the unsteady boundary layer flow and heat transfer past a stretching sheet in a porous medium are analysed by Ishak et al. (2006). A similarity solution for the boundary layer flow through a porous medium over a stretching porous wall is found by Tamayol et al. (2010). Fang and Zhang (2010) revisited the heat transfer over a stretching sheet with mass transfer in a porous medium. The flow and the diffusion of chemically reactive species over a non-linearly stretching sheet immersed in a porous medium are studied by Ziabakhsh et al. (2010). Also a remark on the validity of the Brinkman model in particular on the effective viscosity was made by Auriault (2009).

Mukhopadhyay (2009) studied the unsteady boundary layer flow and heat transfer past a porous stretching sheet with variable viscosity and thermal diffusivity. Pal and Hiremath (2010) studied the computational modeling of heat transfer over an unsteady stretching surface embedded in a porous medium by considering the effects of unit film thickness, viscous dissipation and uniform heat source/sink. Also, Mukhopadhyay (2009) investigated the effects of thermal radiation on unsteady mixed convection flow and heat transfer over a porous stretching surface in a porous medium. Noor and Hashim (2010) studied the effects of thermo-capillarity and the magnetic field in a thin liquid film flow over an unsteady stretching surface by considering non-uniform film thickness.

Nandeppanavar et al. (2010) studied the flow and heat transfer characteristics of a viscoelastic fluid in a porous medium over an impermeable stretching sheet with viscous dissipation. Also, Nandeppanavar et al. (2011) investigated the effects of thermal radiation and non-uniform heat source on heat transfer of MHD viscoelastic fluid flow over a linear stretching sheet. Abel et al. (2010) investigated the flow and heat transfer of a second grade fluid over linear stretching sheet in a porous medium

It is worth mentioning that the stretching need not be linear. In view of this, Kumaran and Ramanaih (1996) studied the flow over a quadratic stretching sheet. Magyari and Keller (1999), Elbashbeshy (2001), Khan and Sanjayanand (2005), Sanjayanand and Khan (2006), Sajjid and Hayat (2008), Partha et al. (2005) studied the heat transfer characteristics of viscous and viscoelastic fluid flows over an exponentially stretching sheet. Vajravelu (2001), Vajravelu and Cannon (2006) are also studied the flow over non-linearly stretching sheet.

There is no heat transfer analysis with thermal radiation and viscous dissipation of a viscous fluid over nonlinear stretching through a porous medium. Hence this work is carried on.

\section{Basic equations and boundary conditions}

Consider the steady flow and heat transfer of a viscous and incompressible fluid past a semi-infinite stretching sheet in the region $y>0$, as shown I Fig.1. Keeping the origin fixed, two equal and opposite forces are suddenly applied along the $x$-axis, which results in stretching of the sheet and hence the flow is generated. Under consideration of the boundary layer approximation, the basic equations of governing flow and heat transfer due to stretching of the sheet are given by

$$
\frac{\partial u}{\partial x}+\frac{\partial v}{\partial y}=0
$$




$$
\begin{aligned}
& u \frac{\partial u}{\partial x}+v \frac{\partial u}{\partial y}=v \frac{\partial^{2} u}{\partial y^{2}}-\frac{v}{k^{\prime}} u \\
& u \frac{\partial T}{\partial x}+v \frac{\partial T}{\partial y}=\alpha \frac{\partial^{2} T}{\partial y^{2}}-\frac{1}{\rho c_{p}} \frac{\partial q_{r}}{\partial y}+\left\{\frac{v}{C_{p}}\left(\frac{\partial u}{\partial y}\right)^{2}+\frac{v}{c_{p} k^{\prime}} u^{2}\right\}
\end{aligned}
$$

Subject to the initial and boundary conditions

$$
\begin{aligned}
& u=\frac{v}{L^{4 / 3}} x^{1 / 3}, \quad v=0 \quad \text { at } \quad y=0, \\
& u \rightarrow 0 \quad \text { as } \quad y \rightarrow \infty, \\
& T=T_{w}=T \infty+A\left(\frac{x}{l}\right)^{m} \text { at } y=0, \\
& T \rightarrow T_{\infty} \quad \text { as } \quad y \rightarrow \infty, \\
& q_{w}=-k\left(\frac{\partial t}{\partial y}\right)_{w}=D\left(\frac{x}{l}\right)^{m} \text { at } y=0, \\
& T \rightarrow \infty \quad \text { as } \quad y \rightarrow \infty
\end{aligned}
$$

where $(x, y)$ denotes the Cartesian coordinates along the sheet and normal to it, $u$ and $v$ are the velocity components of the fluid in the $x$ and $y$ directions, respectively, and $v$ is the kinematic viscosity, $k^{\prime}$ is the permeability of the porous medium. $T$ is the fluid temperature, $\alpha=\frac{k}{\rho c_{p}}$ is the thermal diffusivity, $\rho$ is the density, $C_{p}$ is the specific heat of the fluid at constant pressure and $q_{r}$ is the radiative heat flux, $k_{l}$ is the porous parameter and $l$ is the characteristic length.

Now we introduce the following new variables

$$
\begin{array}{ll}
\eta=y \frac{x^{-1 / 3}}{L^{2 / 3}}, & u=\frac{v}{L^{4 / 3}} x^{1 / 3} f^{\prime}(\eta), \quad \text { and } \quad v=-\frac{v}{L^{2 / 3}} x^{-1 / 3} \frac{2 f-\eta f^{\prime}}{3}, \\
\theta(\eta)=\frac{T-T_{\infty}}{T_{w}-T_{\infty}}, & g(\eta)=\frac{T-T_{\infty}}{(D / k) x^{m+1 / 3} L^{2 / 3-m}} .
\end{array}
$$

Using the initial and boundary conditions (2.4a), (2.4b), (2.4c) and the new variables (2.5) in Eqs (2.2) and (2.3) we have

$$
3 f^{\prime \prime \prime}+2 f f^{\prime \prime}-\left(f^{\prime}\right)^{2}+k_{1} f^{\prime}=0
$$




$$
\begin{aligned}
& \theta^{\prime \prime}+\frac{2 k_{0}}{3} \operatorname{Pr} f \theta^{\prime}-\operatorname{Pr} k_{0} m f^{\prime} \theta=-k_{0} \operatorname{Ec}\left(f^{\prime \prime}\right)^{2}-k_{l} \operatorname{Ec} k_{0}\left(f^{\prime}\right)^{2} \quad(\text { with PST condition), } \\
& g^{\prime \prime}+\frac{2 k_{0}}{3} \operatorname{Pr} f g^{\prime}-\operatorname{Pr} k_{0}\left(m+\frac{1}{3}\right) f^{\prime} g=-\operatorname{Pr} k_{0} \operatorname{Es}\left(f^{\prime \prime}\right)^{2}-k_{0} k_{l} \operatorname{Es} f^{\prime 2} \text { (with PHF condition), }
\end{aligned}
$$

with boundary conditions

$$
\begin{aligned}
& f(0)=0, \quad f^{\prime}(0)=1 \quad f^{\prime}(\infty) \rightarrow 0, \\
& \theta(0)=1, \quad \theta(\infty) \rightarrow 0 \quad \text { (PST), } \\
& g^{\prime}(0)=-1, \quad g(\infty) \rightarrow 0 \quad(\mathrm{PHF})
\end{aligned}
$$

where,

$$
\begin{aligned}
& k_{0}=\frac{3 N r}{(3 N r+4)}, \\
& k_{l}=\left(-\frac{3}{4} L^{4 / 3}\right) \text { porous parameter, } \\
& \operatorname{Pr}=\frac{v}{\alpha}(\text { Prandtl number }), \\
& \mathrm{Ec}=\frac{v^{2} L^{m-8 / 3}}{A c_{p} x^{m-2 / 3}} \text { (Eckert number in PST case), } \\
& \mathrm{Es}=\frac{v^{2} L^{m-10 / 3}}{(D / K) c_{p} x^{m-1 / 3}}(\text { Eckert number in PHF case), } \\
& N r=\frac{k^{*} k}{4 \sigma^{*} T_{\infty}^{3}}(\text { thermal radiation parameter). }
\end{aligned}
$$

Here the prime denotes the differentiation with respect to the similarity variable $\eta$.

The physical quantities of this problem are the skin friction coefficient $C_{f}$ and local Nusselt number $\mathrm{Nu}_{x}$ which are defined as

$$
C_{f}=\frac{\tau_{w}}{\rho u_{w}{ }^{2}}, \quad \mathrm{Nu}_{x}=\frac{x q_{w}}{k\left(T_{w}-T_{\infty}\right)}
$$

where the skin friction $\tau_{w}$ and the heat transfer from the sheet $q_{w}$ are given by 


$$
\begin{aligned}
& \tau_{w}=\mu\left(\frac{\partial u}{\partial y}\right)_{y=0} \text { and } \\
& q_{w}=\left(\frac{\partial T}{\partial y}\right)_{y=0}+\left(q_{r}\right)_{w}=-\frac{k A}{k_{0} L} \theta^{\prime}(0)\left(\frac{x}{L}\right)^{m-1 / 3},
\end{aligned}
$$

with $\mu$ being the dynamic viscosity. Using Eqs (2.4a), (2.4b), (2.4c) in Eqs (2.13) and (2.14), we have

$$
\begin{aligned}
& C_{f}\left(\mathrm{Re}_{x}\right)^{1 / 2}=f^{\prime \prime}(0), \\
& \frac{\mathrm{Nu}_{x}}{\left(\mathrm{Re}_{x}\right)^{1 / 2}}=-\theta^{\prime}(0) \text { for PST case, } \\
& \frac{\mathrm{Nu}_{x}}{\left(\mathrm{Re}_{x}\right)^{1 / 2}}=\frac{1}{g(0)} \text { for PHF case }
\end{aligned}
$$

where $\operatorname{Re}_{x}=\frac{L^{2 / 3} x^{1 / 3}}{v}$ is the local Reynolds's number.

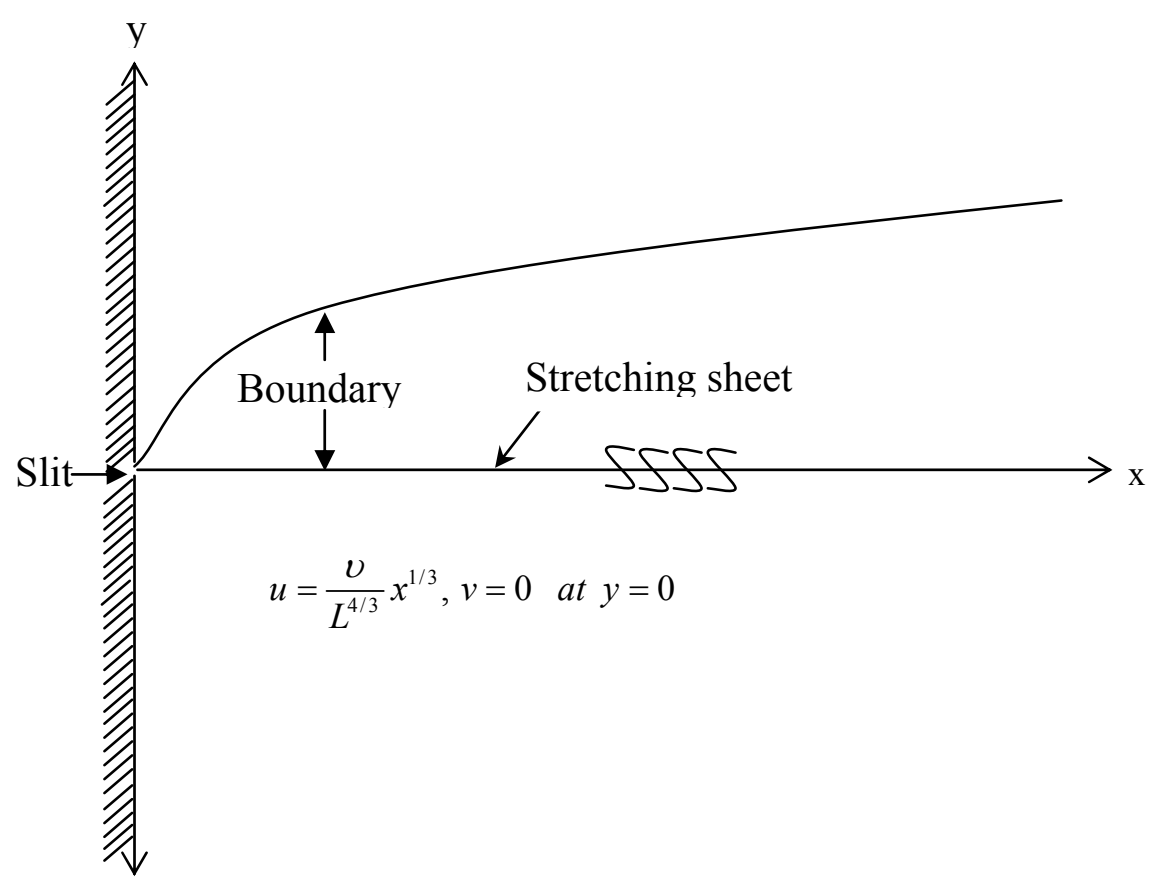

Fig.1. Schematic diagram of non-linear stretching sheet.

\section{Numerical solution of the problem}

The non-linear differential Eqs (2.6)-(2.7) and (2.8) with appropriate boundary conditions given in Eqs (2.9) to (2.11) are solved numerically by the most efficient numerical shooting technique with the fourth order Runge-Kutta algorithm (Please see Cebeci and Bradshaw (1984), Conte and Boor (1972) for more 
details). The non-linear differential equations are first decomposed into a system of first order differential equations in the form

$$
\begin{aligned}
& \frac{d f_{0}}{d \eta}=f_{1}, \\
& \frac{d f_{1}}{d \eta}=f_{2}, \\
& \frac{d f_{2}}{d \eta}=-\frac{2}{3} f_{0} f_{2}+\frac{1}{3} f_{1}^{2}-\frac{1}{3} k_{1} f_{1}, \\
& \frac{d \theta_{0}}{d \eta}=\theta_{1}, \\
& \frac{d \theta_{1}}{d \eta}=-\frac{2}{3} \operatorname{Pr} f_{0} \theta_{1}+\operatorname{Pr} k_{0} m f_{1} \theta_{0}-k_{0} \operatorname{Ec} f_{2}^{2}-k_{0} k_{1} \operatorname{Ec} f_{1}^{2} .
\end{aligned}
$$

Corresponding boundary conditions takes the form,

$$
\begin{aligned}
& f_{0}(0)=0, \quad f_{1}(0)=1, \quad \theta_{0}(0)=1, \\
& f_{1}(\infty)=0, \quad \theta_{0}(\infty)=0
\end{aligned}
$$

where

$$
f_{0}=f(\eta), \quad \text { and } \quad \theta_{0}=\theta(\eta)
$$

The above boundary value problem is first converted into an initial value problem by appropriately guessing the missing slopes. The resulting IVP is solved by the shooting method for several sets of parameters. This is done on a trial and error basis. The step length of $h=0.01$ is employed for the computation purpose. The convergence criterion largely depends on the fairly good guesses of the initial conditions. The iterative process is carried out until the relative difference between the current and the previous iterative values matches up to a tolerance of $10^{-5}$. Once the convergence is achieved we integrate the resultant ordinary differential equations using the standard fourth order Runge-Kutta method to obtain the required solution.

\section{Discussion of the results}

A problem for momentum and heat transfer viscous flow over a non-liner stretching surface in porous media with viscous dissipation and thermal radiation is examined in this paper. The basic boundary layer partial differential equations, which are non-linear, have been converted into a set of non-linear ordinary differential equations by applying suitable similarity transformations and their solutions are obtained numerically by the Runge-Kutta method with shooting technique. The study has been extended for two different heating processes, namely:

(i) prescribed power law surface temperature (PST) and

(ii) prescribed power law heat flux (PHF). 
Figure 2 is plotted for velocity profiles for different values of the porosity parameter $k_{1}$, from this figure we can observe that the velocity profile decreases with an increase of parametric values of the porosity parameter.

Figures $3 a$ and $b$, respectively, reveal that the effect of increasing the values of the local Eckert number $\mathrm{Ec}(\mathrm{Es})$ is to increase temperature distribution in the flow region in both the cases of PST and PHF. This behavior of temperature enhancement occurs as heat energy is stored in the fluid due to frictional heating.

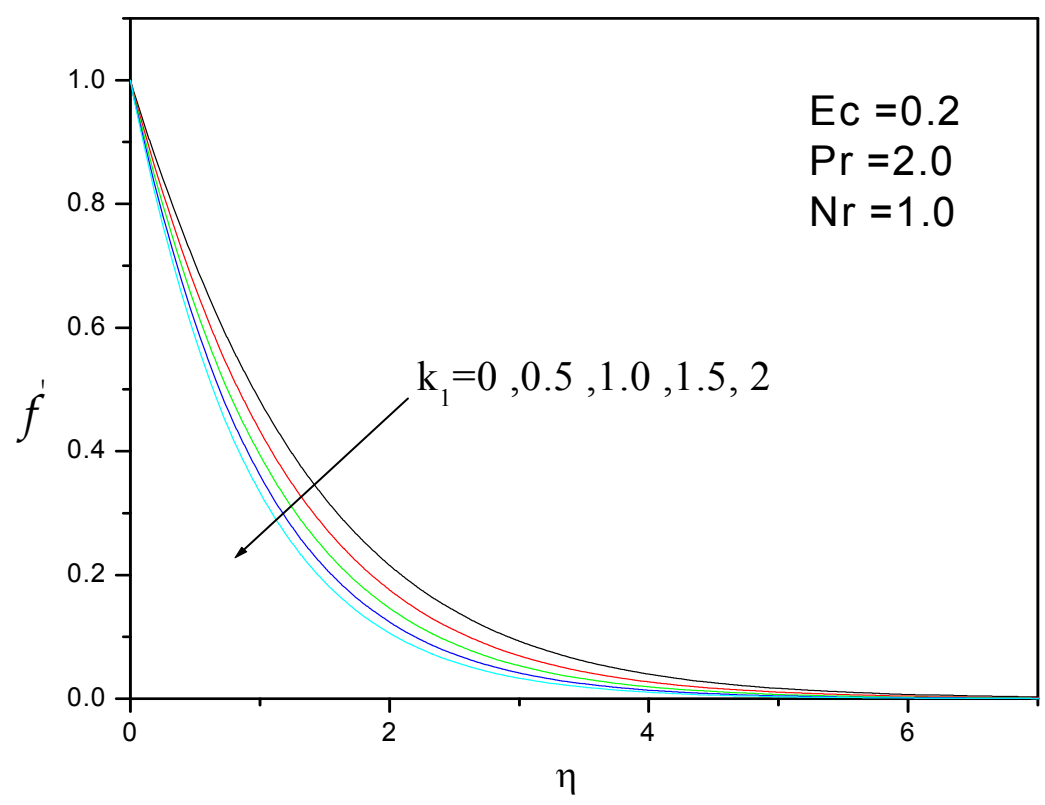

Fig.2. Velocity profile $f^{\prime}$ for different values of $k_{l}$.

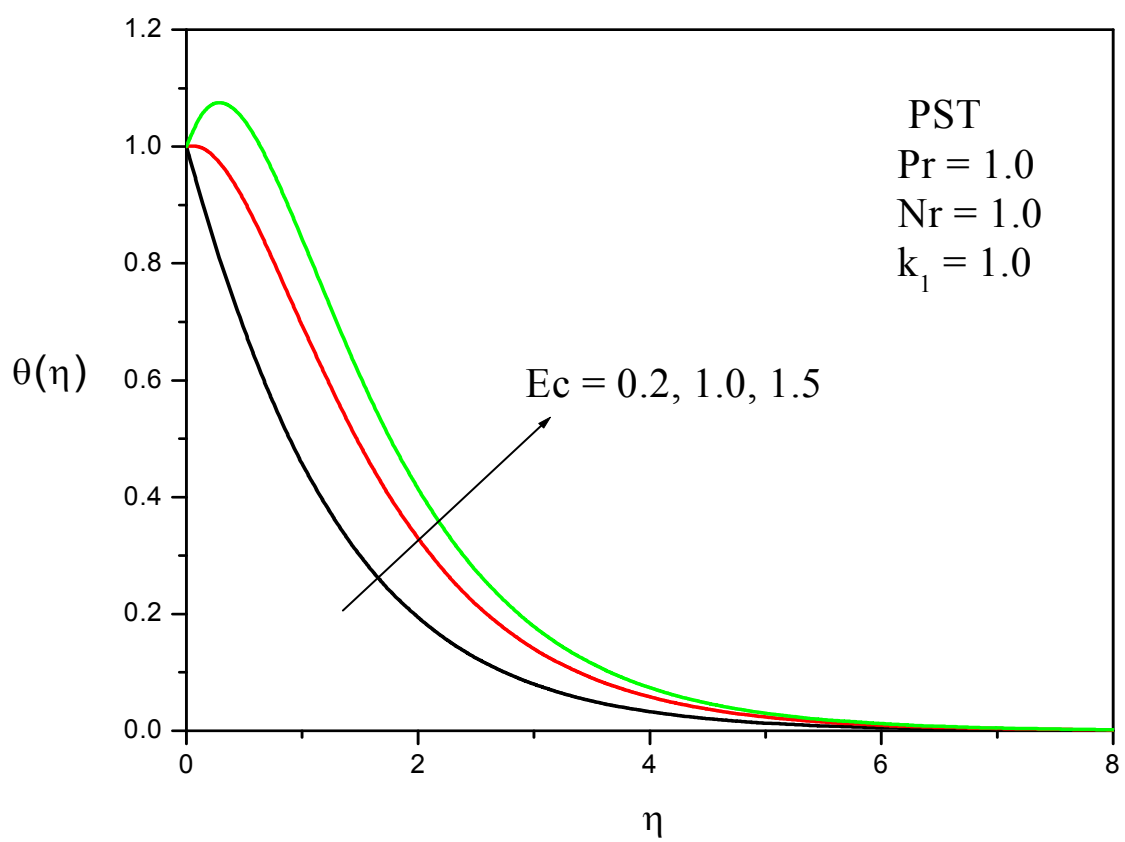

Fig.3a. Temperature profile for various values of Ec. 


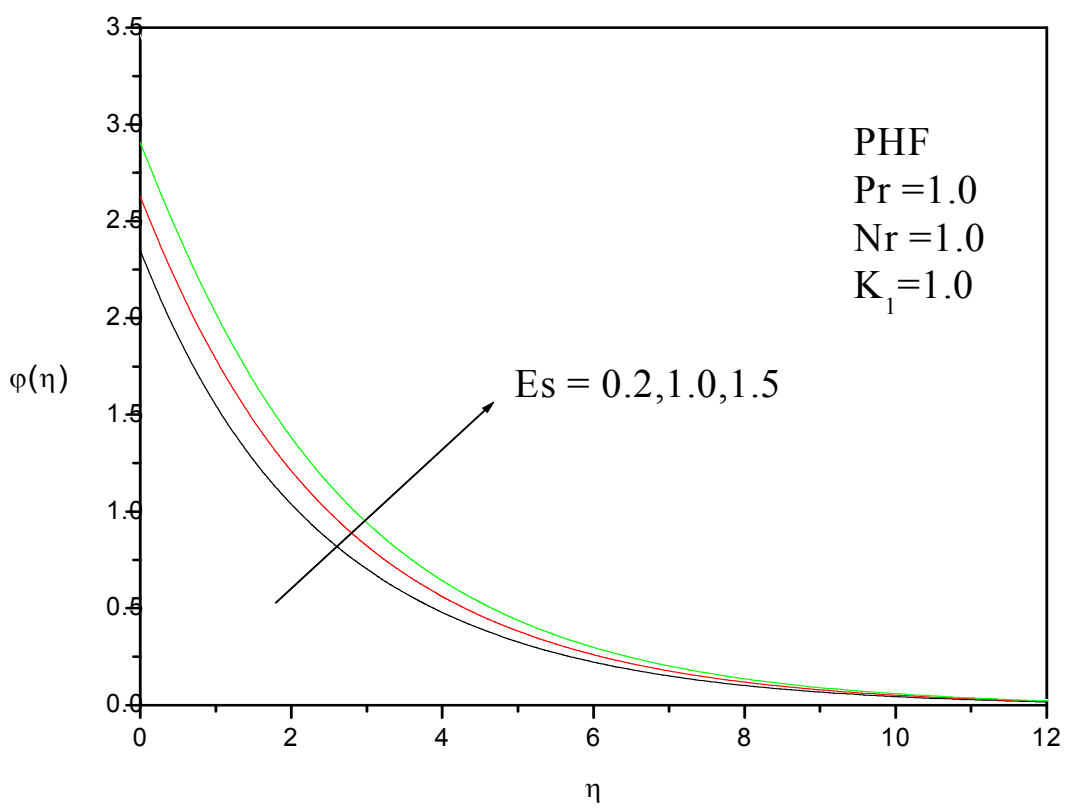

Fig.3b. Temperature profile for various values of Es (PHF case).

Figures $4 \mathrm{a}$ and $4 \mathrm{~b}$ illustrate the variation of temperature profiles for various values of the Prandtl number (Pr). From these figures it is seen that the temperature decreases with increasing the values of the Prandtl number Pr in the boundary layer. From these plots, it is evident that temperature in the boundary layer falls very quickly for large values of the Prandtl number because the thickness of the boundary layer decreases with an increase in the value of the Prandtl number.

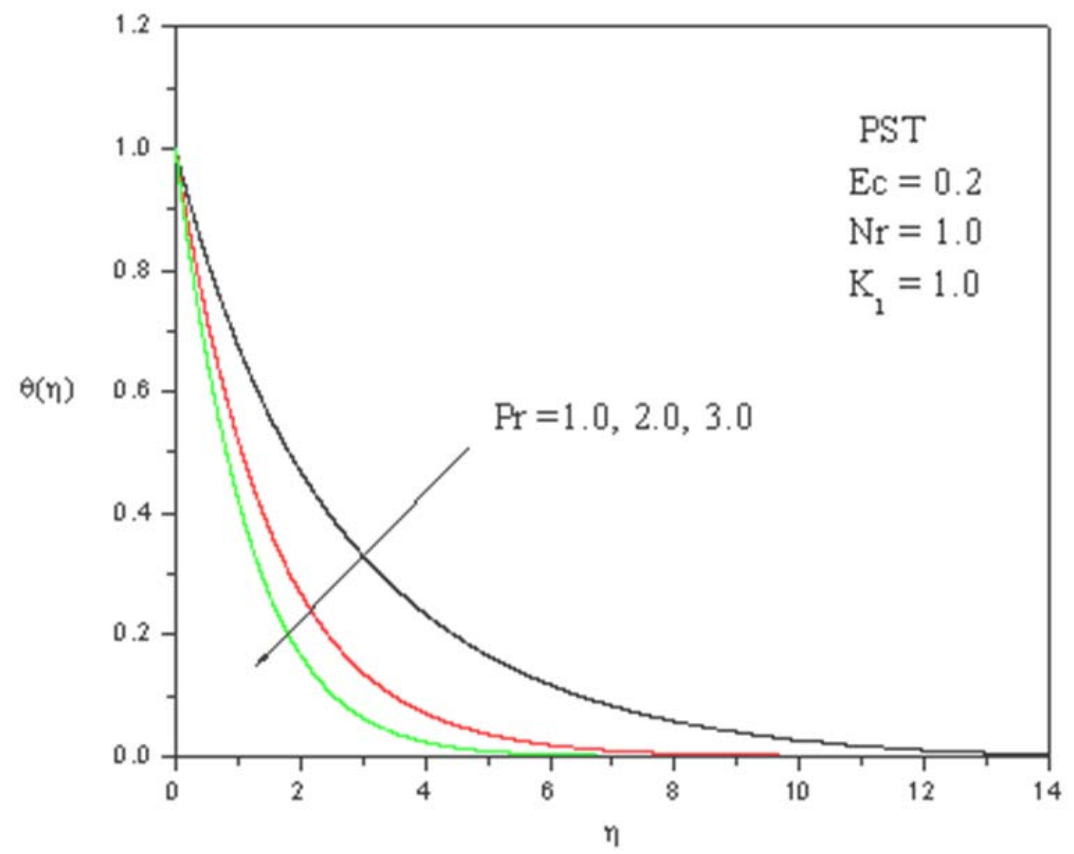

Fig.4a. Temperature profile for various values of $\operatorname{Pr}$ (PST case). 


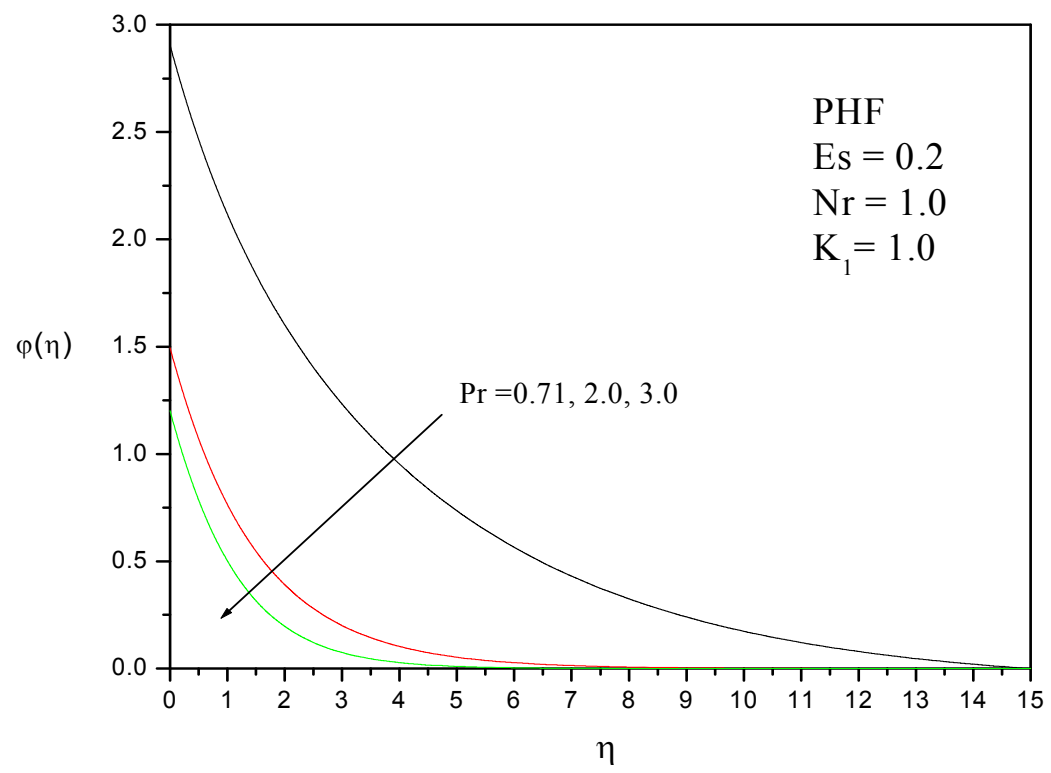

Fig.4b. Temperature profile for various values of $\operatorname{Pr}$ (PHF case).

Figures $5 \mathrm{a}$ and $5 \mathrm{~b}$ are drawn for temperature profiles for PST and PHF cases respectively .From these figures, it is apparent that the temperature is unchanged at the wall with the change of physical parameters in the PST case and we also observe that the temperature increases with an increase in the value of $k_{l}$, in both PST and PHF cases. This is due to the fact that an increase of viscous normal stress gives rise to thickening of the thermal boundary layer.

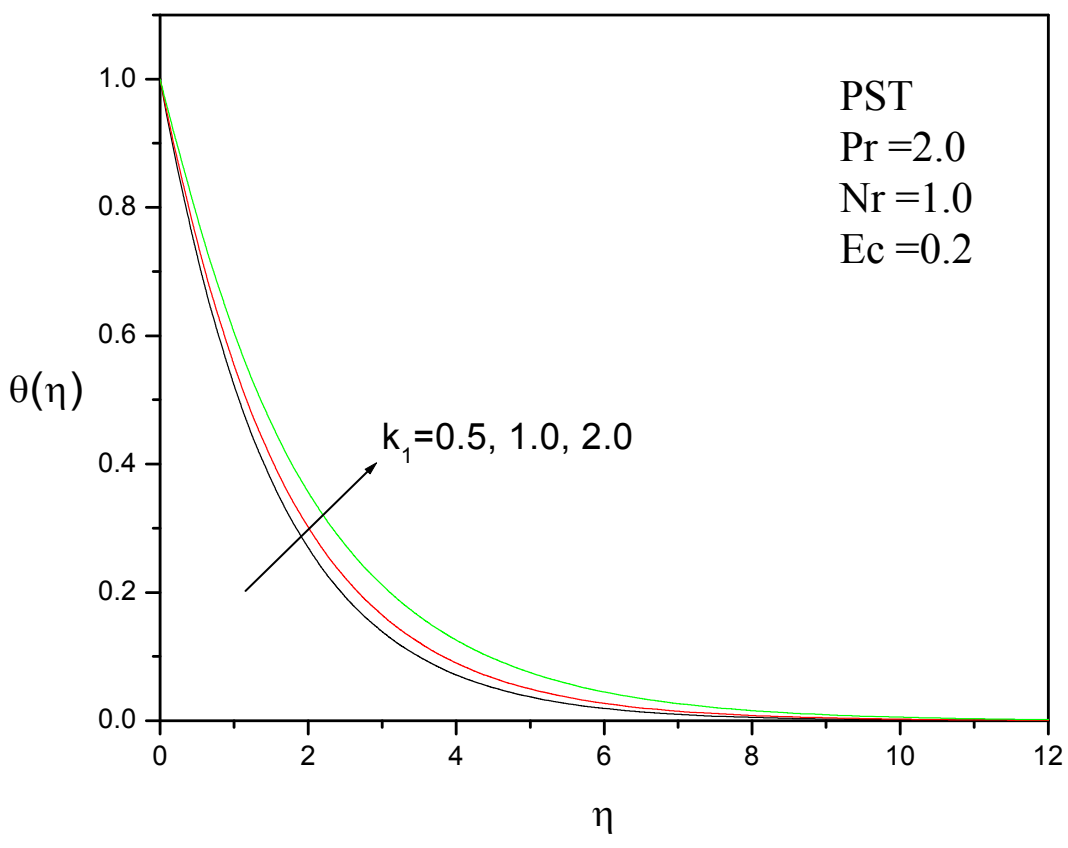

Fig.5a. Temperature profile for various values of $k_{1}$. 


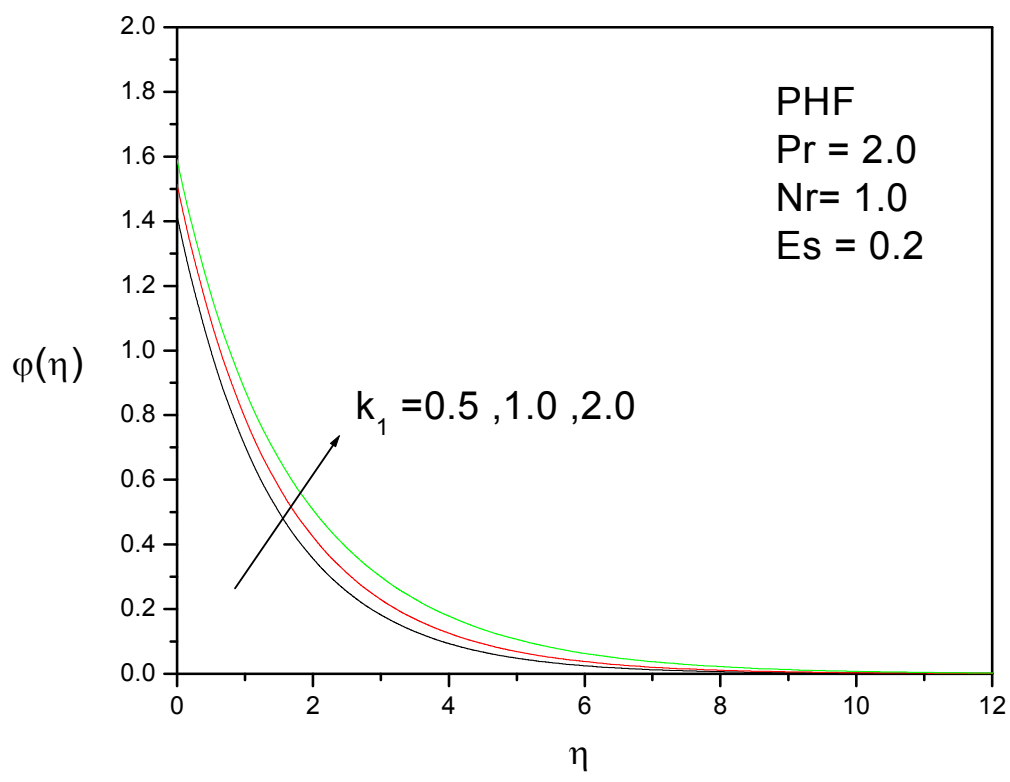

Fig.5b. Temperature profile for various values of $k_{1}$.

Figures $6 \mathrm{a}$ and $6 \mathrm{~b}$ represent the temperature profiles for PST and PHF cases, respectively, for various values of the thermal radiation parameter $N r$. It is observed that an increase in the thermal radiation parameter $(\mathrm{Nr})$ produces a significant increase in the thickness of the thermal boundary and so the temperature distribution increases with increasing values of $\mathrm{Nr}$ in both cases. The effect of $\mathrm{Nr}$ is to enhance the heat. Thus it is pointed out that the radiation should be minimized to have the cooling process at a faster rate.

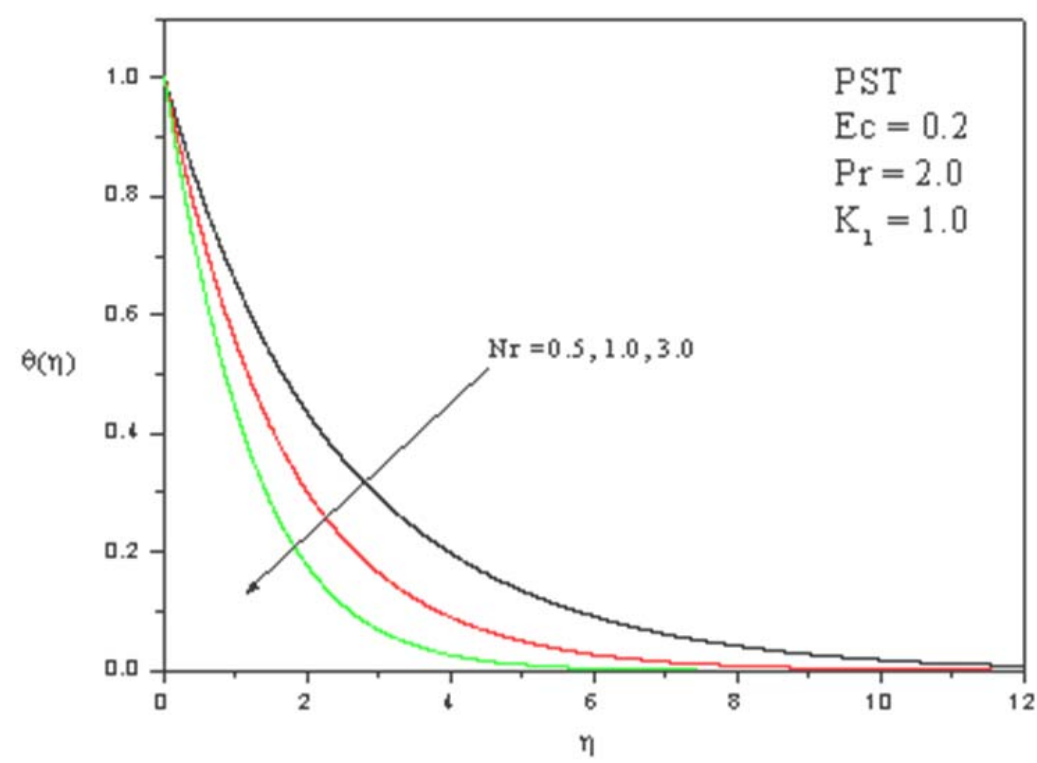

Fig.6a. Temperature profile for various values of $N r$. 


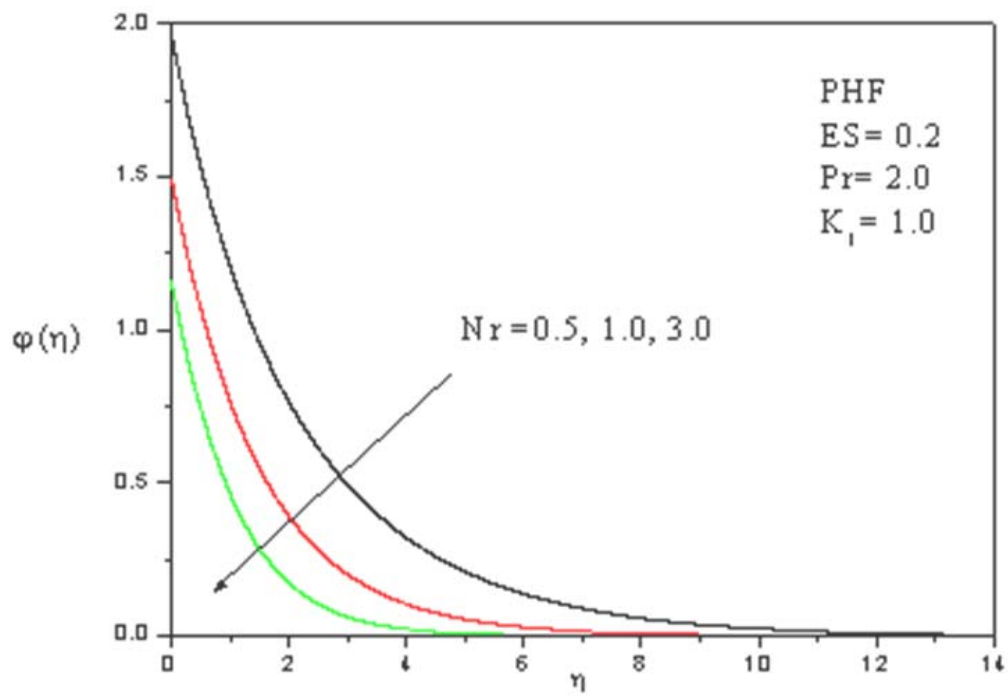

Fig.6b. Temperature profile for various values of $\mathrm{Nr}$.

Table 1. Wall temperature gradient $\left[-\theta^{\prime}(0)\right]$ (PST case with $\left.m=2 / 3\right)$ and wall temperature $\varphi(0)(\mathrm{PHF}$ case with $m=1 / 3$ ) for various values of $p r, \operatorname{Tr}$ and $\operatorname{Ec}(\operatorname{Es})$ at different values of $k_{1}$.

\begin{tabular}{|c|c|c|c|c|}
\hline \multirow{2}{*}{$\operatorname{Pr}$} & \multirow{2}{*}{$\mathrm{Nr}$} & \multirow{2}{*}{$\mathrm{Ec}(\mathrm{Es})$} & PST & PHF \\
\hline & & & $-\theta^{\prime}(0)$ & $\varphi(0)$ \\
\hline & & & \multicolumn{2}{|c|}{$K=0.0$} \\
\hline & 0.5 & & 0.535936 & 1.757780 \\
\hline \multirow[t]{3}{*}{2} & 1 & 0.2 & 0.713899 & 1.312961 \\
\hline & 3 & & 0.947651 & 0.982945 \\
\hline & 7 & & 1.057013 & 0.878837 \\
\hline 0.71 & & & 0.359562 & 2.634675 \\
\hline 2 & 1 & 0.2 & 0.713899 & 1.312961 \\
\hline 3 & & & 0.908171 & 1.028721 \\
\hline \multirow[t]{2}{*}{10} & & & 1.742119 & 0.525615 \\
\hline & & 0 & 0.973974 & 1.26721 \\
\hline \multirow[t]{2}{*}{3} & 1 & 0.2 & 0.908171 & 1.30722 \\
\hline & & 1.0 & 0.644959 & 1.56627 \\
\hline
\end{tabular}


Table 2. Temperatures $\theta(\eta)$ and $\varphi(\eta)$ when $\operatorname{Pr}=2$ and $T_{r}=3$ for several values of $m$.

\begin{tabular}{cccccc}
\hline & & \multicolumn{3}{c}{$k_{1}=\mathbf{0 . 5}$} & \multicolumn{3}{c}{$k_{l}=\mathbf{0 . 5}$} \\
\cline { 3 - 6 }$m$ & $\eta$ & $\theta(\eta)$ & $-\theta^{\prime}(0)$ & $\varphi(\eta)$ & $-\varphi(\eta)$ \\
\hline 0 & 0 & 1.0 & 0.879564 & 1.075900 & 1.000000 \\
1 & 0 & 0.751732 & 1.0 & 1.0 & 1.296053 \\
3 & 0 & 1.000000 & 2.004106 & 0.496324 & 1.000000 \\
\hline
\end{tabular}

\section{Acknowledgements}

One of the authors (MMN.) gratefully acknowledges the financial support of University Grants Commission, New-Delhi, India for pursuing this work under major research project (Grant F.No:39-59/2010 (SR).

\section{Nomenclature}

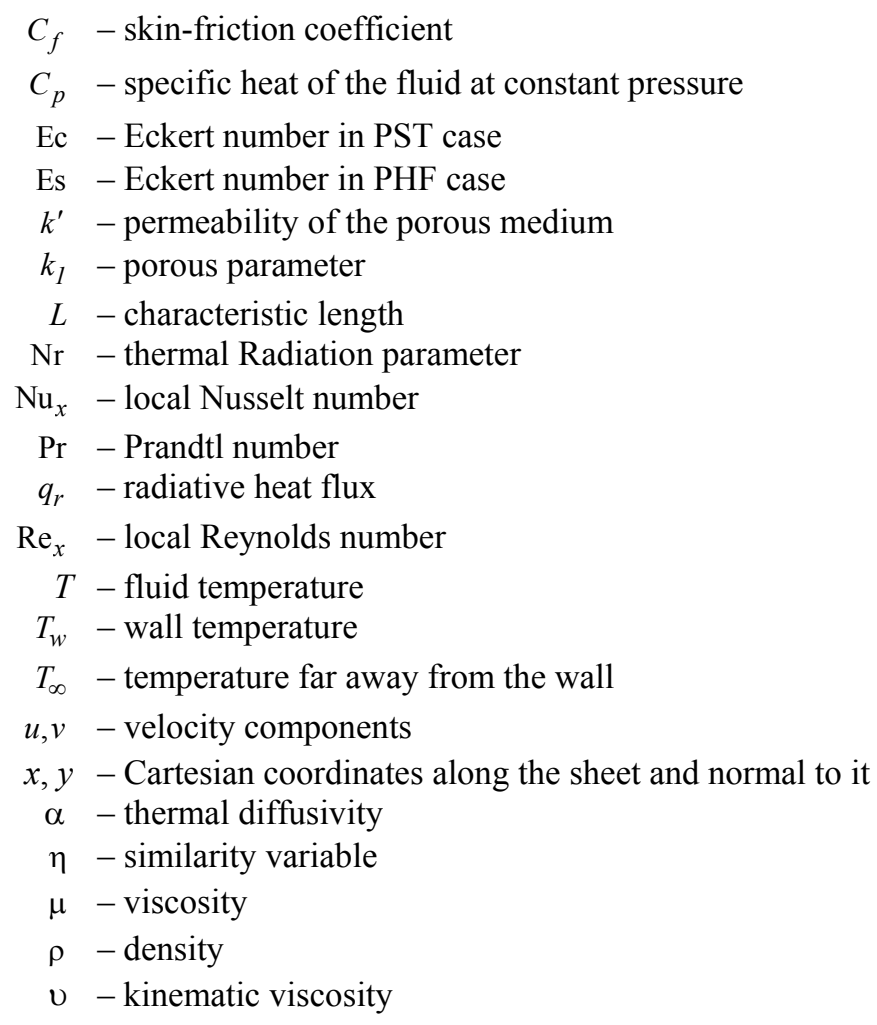

\section{References}

Abel M.S, Mahantesh M. Nandeppanavar and Malipatil S.B. (2010): Heat transfer in a second grade fluid through porous medium from a permeable stretching sheet with non-uniform heat source/sink. - Int. J. Heat and Mass Transfer, vol.53, pp.1788-1795.

Auriault J.L. (2009): On the domain of validity of Brinkman's equation. - Transp. Porous Med., vol.79, pp.215-223. 
Carragher P. and Crane L.J. (1982): Heat transfer on a continuous stretching sheet. - Z. Angew. Math. Mech., vol.62, pp.564-565.

Cebeci T. and Bradshaw P. (1984): Physical computational aspects of convective heat transfer. - New York: SpringerVerlag.

Crane L.J. (1970): Flow past a stretching plate. - Z. Angew. Math. Phys., vol.21, pp.645-647.

Conte S.D. and De. Boor C. (1972): Elementary Numerical Analysis. - New York: McGraw-Hill.

Elbashbeshy E.M.A. (2001): Heat transfer over an exponentially stretching continuous surface with suction. - Arch. Mech., vol.53, pp.643-651.

Fang T. and Zhang J. (2010): Note on the heat transfer of flows over a stretching wall in porous media: exact solutions. - Transp. Porous Med., doi: 10.1007/s11242-010-9640-6.

Gupta P.S. and Gupta A.S. (1977): Heat and mass transfer on a stretching sheet with suction or blowing. - Can. J. Chem. Eng., vol.55, pp.744-746.

Ishak A., Nazar R. and Pop I. (2006): Steady and unsteady boundary layers due to a stretching vertical sheet in a porous medium using Darcy-Brinkman equation model. - Int. J. Appl. Mech. Eng., vol.11, pp.623-637.

Khan S.K. and Sanjayanand S.K. (2005): Viscoelastic boundary layer flow and heat transfer over an exponential stretching sheet. - Int. J. Heat Mass Transfer, vol.48, pp.1534-1542.

Kumaran V. and Ramanaih G. (1996): A note on the flow over stretching sheet. - Arch. Mech., vol.116, pp.229-233.

Liu I.C. (2005): Exact solutions for a fluid-saturated porous medium with heat and mass transfer. - J. Mech., vol.21, pp.57-62.

Liu I.C. (2006): Flow and heat transfer of viscous fluids saturated in porous media over a permeable non-isothermal stretching sheet. - Transp. Porous Med., vol.64, pp.375-392.

Mahantesh M. Nandeppanavar, Abel M.S. and Vajravelu K. (2010): Flow and heat transfer characteristics of viscoelastic fluid in a porous medium over an impermeable stretching sheet with viscous dissipation. - Int. J. Heat and Mass Transfer, vol.53, pp.4707-4713.

Mahantesh M. Nandeppanavar, Vajravelu K. and Abel M.S. (2011): Heat transfer in MHD viscoelastic boundary layer flow over a stretching sheet with thermal radiation and non-uniform heat source/sink. - Communications in NonLinear science and Numerical Simulations (Article in press).

Magyari E. and Keller B. (1999): Heat mass transfer in the boundary layers on an exponentially stretching sheet. - J. Phys. D: Appl. Phys., vol.32, 577-585.

Mukhopadhyay S. (2009): Unsteady boundary layer flow and heat transfer past a porous stretching sheet in presence of variable viscosity and thermal diffusivity. - Int. J. Heat Mass Transfer, vol.52, pp.5213-521.

Mukhopadhyay S. (2009): Effect of thermal radiation on unsteady mixed convection flow and heat transfer over a porous stretching surface in porous medium. - Int. J. Heat Mass Transfer, vol.52, pp.3261-3265.

Noor N.F.M. and Hashim I. (2010): Thermocapillary and magnetic field effects in a thin liquid film on an unsteady stretching surface. - Int. J. Heat Mass Transfer, vol.53, pp.2044-2051.

Pal D. and Hiremath P.S. (2010): Computational modeling of heat transfer over an unsteady stretching surface embedded in a porous medium. - Acta Mech. DOI10.1007/s11012009-9254-7.

Partha M.K., Murthy P.V.S.N. and Rajasekhar G.P. (2005): Effect of viscous dissipation on the mixed convection of heat transfer from an exponential stretching surface. - Heat Mass Transfer, vol.41, pp.360-366.

Sajid M. and Hayat T. (2008): Influence of thermal radiation on the boundary layer flow due to an exponentially stretching sheet. - Int. Comm. Heat Mass Transfer, vol.35, pp.347-356.

Sakiadis B.C. (1961): Boundary layer behavior on continuous solid surface. - AIChE J 7, pp.26-28.

Sanjayanand E. and Khan S.K. (2006): On heat and mass transfer in viscoelastic boundary layer flow over an exponentially stretching sheet. - Int. J. Therm. Sci., vol.45, pp.819-828. 
Tamayol A., Hooman K. and Bahrami M. (2010): Thermal analysis of flow in a porous medium over a permeable stretching wall. - Transp. Porous Med. doi:10.1007/s11242-010-9584-X.

Vajravelu K. (2001): Viscous flow over a non-linearly stretching sheet. - Appl. Math. Comput., vol.124, pp.281-288.

Vajravelu K. and Cannon J.R. (2006): Fluid flow over a non-linearly stretching sheet. - Appl. Math. Comput., vol.181, pp.609-618.

Ziabakhsh Z., Domairry G., Bararnia H. and Babazadeh (2010): Analytical solution of flow and diffusion of chemically reactive species over a nonlinearly stretching sheet immersed in a porous medium. - J. Taiwan Inst. Chem. Eng., vol.41, pp.22-28.

Received: December 25, 2011

Revised: November 2, 2012 